\title{
Self-healing of steel corrosion in a model alkaline medium: electrochemical response and surface analysis
}

\author{
D. A. Koleva ${ }^{1}$, J. Hu ${ }^{2}$, H. Kolev ${ }^{3} \&$ K. Van Breugel ${ }^{1}$ \\ ${ }^{1}$ Delft University of Technology, The Netherlands \\ ${ }^{2}$ South China University of Technology, PR China \\ ${ }^{3}$ Bulgarian Academy of Sciences, Institute of Catalysis, Bulgaria
}

\begin{abstract}
The application of polymeric nano-particles for corrosion control in reinforced cement-based systems was studied. As an initial investigation, steel electrodes (St37) were tested in modified cement extract (CE), which is a model pore solution of pH 12.9 and chemical composition: $201 \mathrm{mg} / \mathrm{l} \mathrm{Ca} ; 3.85 \mathrm{mg} / 1 \mathrm{~K} ; 1.33 \mathrm{mg} / \mathrm{l} \mathrm{Na}$; $4 \mathrm{mg} / \mathrm{l} \mathrm{Al}, \mathrm{Fe}<1 \mathrm{mg} / \mathrm{l}$. Corrosion initiating factor was 5\% NaCl. $\mathrm{PEO}_{113}-\mathrm{b}-\mathrm{PS}_{780}$ vesicles at 0.0024 wt. $\%$ in the CE were employed. These are nano sacs of $220 \mathrm{~nm}$, enclosing a volume with a thin membrane, thus able to effectively entrap water-soluble compounds. Ion binding (bulk liquid medium), steel surface adsorption and/or participation in oxide layers formation were the expected mechanisms of particles' involvement. Both "empty" and "Ca-containing" vesicles were studied to evaluate "barrier" effects only vs. self-healing mechanisms. Increased steel corrosion resistance in the presence of both vesicle types was observed, however, it was more pronounced when $\mathrm{Ca}$-containing ones were involved. Cyclic voltammetry and X-ray photoelectron spectroscopy analysis substantiate the self-healing phenomena, through direct evidence of pitting formation and propagation and consecutive healing (and no further pit formation) when Ca-containing vesicles are present.

Keywords: corrosion, cement extract, vesicles, self-healing.
\end{abstract}




\section{Introduction}

The alkaline nature of concrete $(\mathrm{pH}=12.6-13.5)$ generally provides a physicochemical barrier against corrosion of the embedded steel and assures the formation of a stable passive layer on the steel surface [1]. However, corrosion is initiated due to carbonation $(\mathrm{pH}$ drop $<9)$ or chloride contamination $(\mathrm{pH}$ drop $<5$ ) [2-8], resulting in corrosion propagation, accelerated damage and rapid failure [9]. Various protection methods and techniques have been investigated and are successfully applied, e.g. protective coatings/sealers [10,11]; polymer coatings and cathodic protection (CP) (the latter combination leading to increased current demand/de-bonding [12] and possible hydrogen embrittlement or bond-strength degradation [13-18]); concrete re-alkalization [19]; corrosion inhibitors (quickly exhausted and with none or even negative effects on the bulk cement-based matrix [19-21]). It is well known that each method/technique has its limitations and side effects. Moreover, there is no technique that considers both steel reinforcement and cement-based bulk matrix, aiming at improved properties of both materials and superior global performance of reinforced concrete as a system. To this end, in the frame of a novel approach and (initially) within the IOP Self-Healing project SHM08743, executed in TU Delft and financially supported by Agentschap, NL, tailor-made polymeric nano-materials were studied. The aim was to increase steel corrosion resistance via self-healing of corrosion damage, along with improved microstructural characteristics.

As far as the cement-based bulk matrix properties were concerned, the suggested hybrid core-shell nano-particles already proved their positive effect, for example: in the presence of polyethylene oxide (PEO) 113 -b- polystyrene $(\mathrm{PS})_{218}$ micelles (50 nm size) of only $0.025 \mathrm{wt} . \%$ per cement weight, the porosity of the micelle-modified matrix was reduced two times, whereas water permeability was reduced by six orders of magnitude; a more uniform distribution of calcium-silicate-hydrate (C-S-H) and enhanced mechanical properties, but undisturbed water balance was evident from nano-indentation tests [22-25]. For establishing the effects on corrosion resistance, the aforementioned micelles, as well as stabilized (kinetically frozen) $\mathrm{PEO}_{113}-\mathrm{b}-\mathrm{PS}_{760}$ vesicles $(220 \mathrm{~nm}$ size), "empty" and Ca-containing ones, were studied [26-28]. The aim of these studies was to investigate the possibility for maintained positive effects on the cement-based microstructure, along with increased corrosion resistance and self-healing (or repair) of corrosion damage, resulting from Ca-core release. Initial tests were performed in model pore solutions, prior to studies in reinforced mortar and concrete. This paper briefly presents some main outcomes from the investigation on corrosion resistance of St37 steel electrodes in cement extract, modified with the above-defined vesicles [28]. Further, this work substantiates improved corrosion resistance and evidence of self-healing effects through correlating electrochemical performance and steel surface analysis. 


\section{Materials}

\subsection{Polymeric vesicles}

Vesicles were prepared via dialysis from $\mathrm{PEO}_{113}-\mathrm{b}-\mathrm{PS}_{760}$ block-polymer [24-28]. In order to distinguish between "barrier" effects and "self-healing" due to Ca-release, both "empty" and Ca-containing vesicles were used. Their hydrodynamic radius was approximately $220-250 \mathrm{~nm}$, identified by dynamic light scattering (DLS) measurement and transmission electron microscopy (TEM) (Fig. 1).

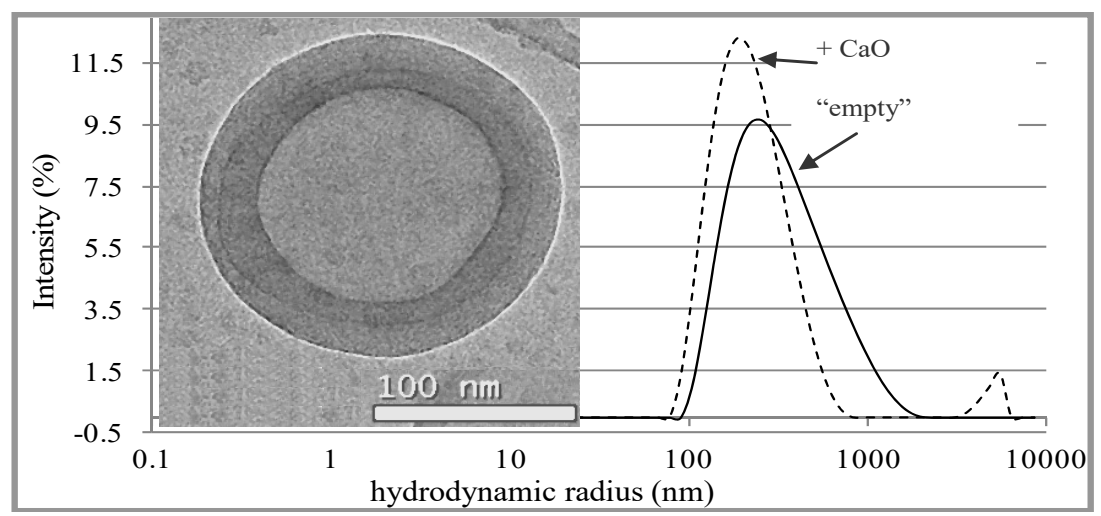

Figure 1: DLS and TEM of vesicles.

\subsection{Cement extract}

As a model, pore solution was prepared by mixing ordinary Portland cement CEM I $42.5 \mathrm{~N}$ and tap water in 1:1 ratio; after $24 \mathrm{~h}$ rotation, the suspension was filtrated and this solution was used as a model medium. The $\mathrm{pH}$ of the cement extract (CE) is 12.6-2.9, the chemical composition, derived via inductive coupled plasma spectrometry (ICP) was: $\mathrm{Ca}-201 \mathrm{mg} / \mathrm{l} ; \mathrm{K}-3.85 \mathrm{mg} / \mathrm{l} ; \mathrm{Na}-1.33 \mathrm{mg} / \mathrm{l}$; $\mathrm{Al}-4 \mathrm{mg} / \mathrm{l}$ and $\mathrm{Fe}<1 \mathrm{mg} / \mathrm{l} ; \mathrm{NaCl}$ was corrosion-inducing factor, added in a concentration of $10 \mathrm{~g} / 1$ to the $\mathrm{CE}$. The aqueous solution of $\mathrm{PEO}_{113}-\mathrm{b}-\mathrm{PS}_{760}$ vesicles was added to $\mathrm{CE}$, thus the vesicles concentration was 0.0024 wt. \% in chloride-free and chloride-containing $\mathrm{CE}$.

\subsection{Steel electrodes}

Low-carbon steel St37 with surface area of $4 \mathrm{~cm}^{2}$ was used. All electrodes were equally treated (grinding, polishing, acetone cleaning and water rinsing) prior to treatment in the relevant solutions and electrochemical measurements. Three 
replicates per condition were tested. Sample designation is as follows: group $\mathrm{CE}$ - steel in CE only; CEn - steel in chloride-containing CE; CEV and CEVC stand for steel in CE modified with "empty" and "Ca-containing" vesicles, whereas CEVn and CEVCn stand for the respective corroding cells.

\section{Methods}

\subsection{Electrochemical measurements}

Autolab-Potentiostat PGSTAT 30, combined with FRA2 module using GPES and FRA interface, was used. Linear polarization resistance (LPR), potentio-dynamic polarization (PDP), impedance spectroscopy (EIS) and cyclic voltammetry (CVA) were employed, using a common 3-electrode cell arrangement (saturated calomel electrode, SCE, as reference electrode). EIS was performed at open circuit potential (OCP) in the frequency range of $50 \mathrm{kHz}$ to $10 \mathrm{mHz}$ by superimposing an ac voltage of $10 \mathrm{mV}$; LPR used a polarization window of $\pm 20 \mathrm{mV}$ vs. OCP; PDP was in the range of $-0.2 \mathrm{~V}$ to $+1.0 \mathrm{~V}$ vs. OCP at a scan rate $0.5 \mathrm{mV} / \mathrm{s}$; CVA scans were in the potential region of $-1.4 \mathrm{~V}$ to $0.6 \mathrm{~V}$ at a scan rate of $5 \mathrm{mV} / \mathrm{s}$ (all electrochemical measurements are as generally employed for the systems under study [22-27]).

\subsection{Surface analysis}

Morphology and composition of the product layers were investigated using scanning electron microscopy (SEM), coupled with energy dispersive X-ray (EDX) analysis as a qualitative and semi quantitative technique, using ESEM Philips XL 30. X-ray photoelectron spectroscopy (XPS) was carried out in the UHV chamber of an electron spectrometer ESCALAB-MkII VG Scientific with a base pressure of about $1 \times 10^{-10} \mathrm{mbar}$ (during the measurement $1 \times 10^{-9} \mathrm{mbar}$ ). The photoelectron spectra were obtained using unmonochromatized Al Ka (hv $=1486.6 \mathrm{eV}$ ) radiation. Passing through $6 \mathrm{~mm}$ slit (entrance/exit) of a hemispherical analyser, electrons with energy $20 \mathrm{eV}$ are detected by a channeltron. The instrumental resolution measured as the full width at a half maximum (fwhm) of the $\mathrm{Ag} 3 \mathrm{~d} 5 / 2$, photoelectron peak is about $1 \mathrm{eV}$. The energy scale is corrected to the $\mathrm{C} 1 \mathrm{~s}$ (peak maximum at $285 \mathrm{eV}$ ) for electrostatic sample charging. The fitting of the recorded XPS spectra was performed, using a symmetrical Gauss-Lorentzian curve fitting after Shirley-type subtraction of the background. Except scanning the steel surface after 7 days, i.e. product layers formed on the steel electrodes in each relevant condition, four-times consecutive mechanical polishing of equal pressure and time duration was performed in depth of the layer in the vacuum chamber of the spectrometer. This technique was chosen to avoid influence on the chemistry of the formed layers, which would otherwise be relevant if Ar sputtering was used. The XPS measurements thus resulted in qualifying and quantifying the composition of the product layers in depth, i.e. for each specimen, four in depth scan results were recorded. 


\section{Results and discussion}

\subsection{Electrochemical measurements}

\subsubsection{OCP evolution and polarization resistance}

A general perception is that the evolution of OCP determines the time to corrosion initiation. For reinforced concrete and alkaline medium, as the here used CE, the steel surface is considered to be passive if OCP is equal or more anodic than $-270 \mathrm{mV} \mathrm{SCE}$. When chlorides (or other corrosion-inducing factors) are present, passivity is lost and localised corrosion initiated - OCP readings shift to more cathodic (more negative) values in this case. Fig. 2 depicts OCP evolution for the control (non-corroding) cases (dotted notation), Fig. 3 shows these values for the corroding cases. Additionally, Figs. 2 and 3 depict the derived (via linear regression) polarization resistance values $\left(R_{p}\right)\left(R_{p}\right.$ in global terms corresponds to corrosion resistance). As can be observed, OCP values fall in the region of passivity and shift towards more noble potentials at the end of the test. These correspond to the gradual increase of $R_{p}$ values, the highest being for the steel treated in "empty" vesicles containing CE. Since there is no corrosion accelerator in the medium for the control samples, only gradual passivation is expected rather than any pronounced effect of the Ca-rich inner "core" of the Ca-containing vesicles. However, adsorption of both vesicle types on the steel surface and their incorporation in the passive layer could result in possible alterations in compactness and/or speed of layer formation. Obviously, barrier effects in terms of diffusion limitations from the adsorbed "empty" vesicles are relevant for specimen CEV, since OCP values are more cathodic, whereas $R_{p}$ values are the highest. This does not necessarily mean a better corrosion resistant layer, but possibly limited oxygen access and hence locally impeded growth of a more inhomogeneous layer. In contrast, the $\mathrm{CE}$ and CEVC specimens exhibit similar $R_{p}$ values.

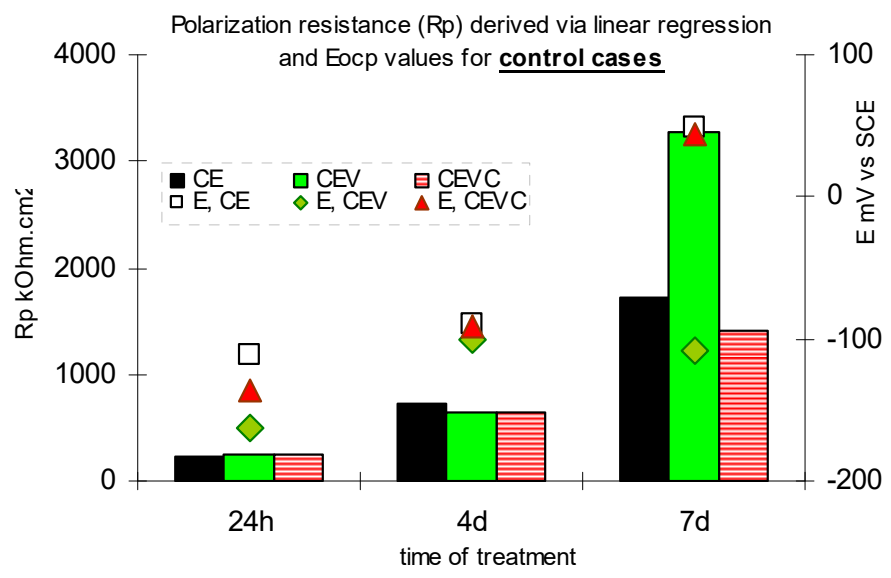

Figure 2: OCP and Rp values for control cases. 
Apparently, the Ca-containing vesicles either act as "nucleation sites" on the steel surface, or adsorb on active spots or both and therefore do not result in diffusion limitations as in the case of CEV specimens.

For the corroding cases (Fig. 3), a shift to more active (cathodic) OCP values is as expected and as observed for specimens CEn (steel treated in vesicles-free solutions). This corresponds to the decreasing $R_{p}$ values with time of treatment. In contrast, specimens CEVn and CEVCn depict OCP values in the passive range at the end of the test (more anodic than $-200 \mathrm{mV}$ ) and higher $R_{p}$ values. The influence of Ca-containing vesicles is here already well pronounced: after initially lower values, and even lowest after 4 days treatment, the $R_{p}$ values for specimens CEVCn depict a significant increase at the end of the test. Since the steel electrodes, treated in CE containing "empty" vesicles do not show this trend (specimens CEVn), the only plausible explanation is that the Ca-containing vesicles exert a "self-repair" or "self-healing" of the already induced corrosion damage on later stages of treatment. These observations are actually confirmed by EIS, PDP and CVA tests and supported by surface analysis.

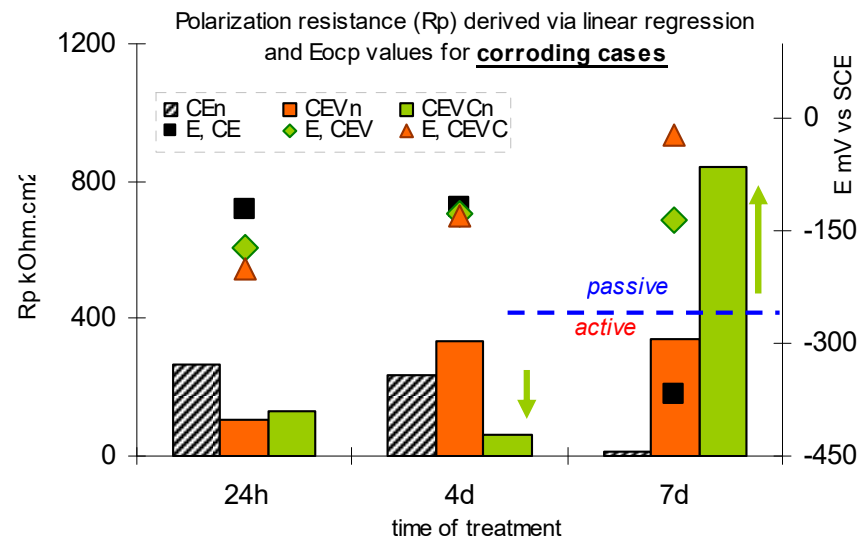

Figure 3: OCP and Rp values for corroding cases.

\subsubsection{EIS, PDP and CVA results}

The EIS response is not subject to elaborated discussion in this paper, but is presented as a direct visualisation of the electrochemical behaviour of the steel electrodes. Fig. 4(a) presents the response for the control specimens after 7 days. Figs. 4(b)-(d) depict the response of all corroding cases after $24 \mathrm{~h}$ (b), 4-day (c) and 7-day (d) treatment. As seen from the plots, the magnitude of impedance $|Z|$ is higher for the steel treated in vesicles containing solutions at all time intervals, accompanied by a mostly constant phase angle of approx. $80^{\circ}$, whereas for the vesicles-free cases the phase angle drops to approx. $65^{\circ}$ at the end of the test. The EIS results clearly show the "self-repair" of the product layer on the steel surface when Ca-containing vesicles are involved: specimens CEVCn depict a response with two time constants after $24 \mathrm{~h}$ treatment, meaning a pronounced 

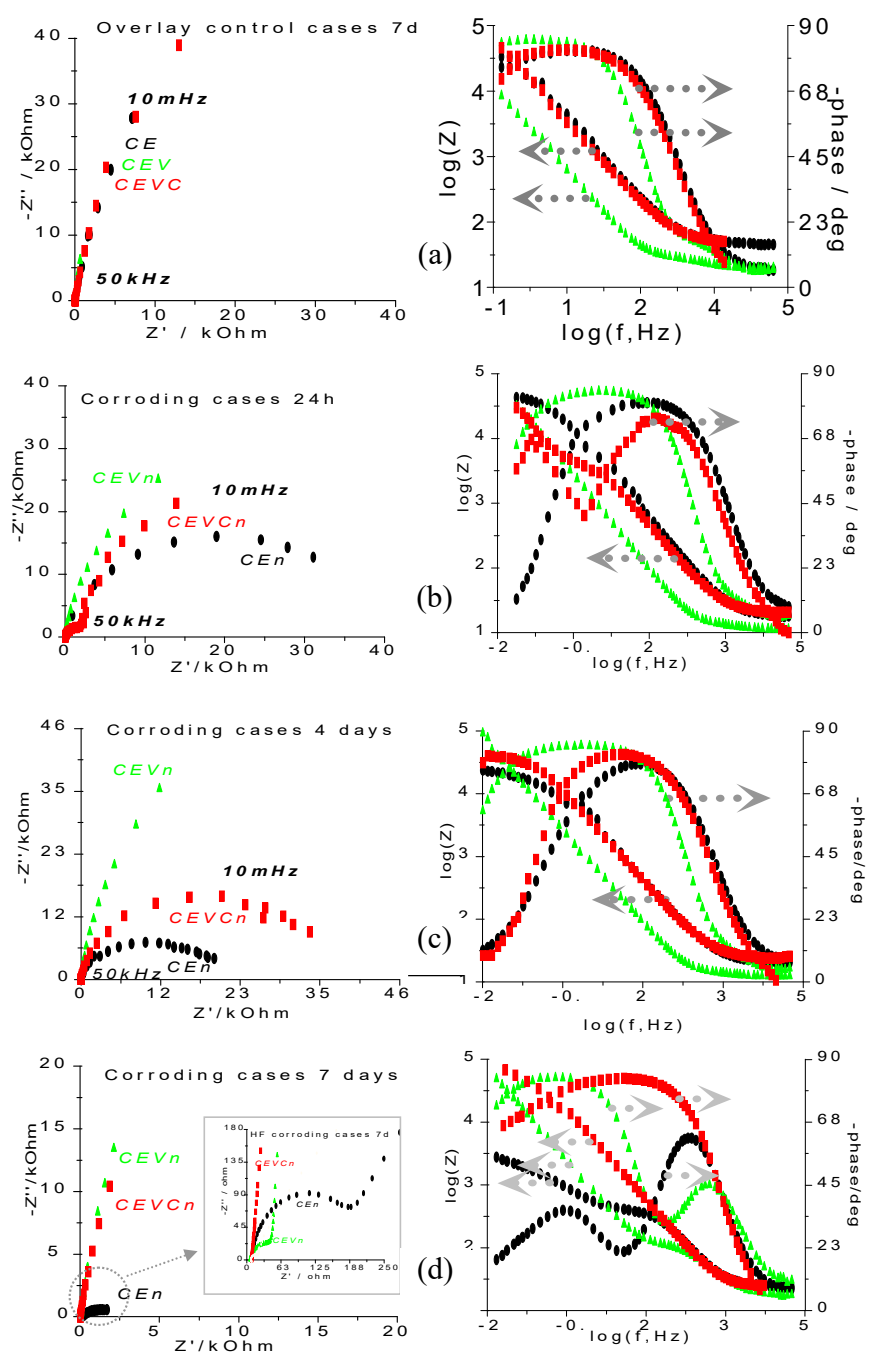

Figure 4: 7-day EIS response for CE, CEV and CEVC (a) and CEn, CEVn, CEVCn after $24 \mathrm{~h}$ (b), 4 days (c) and 7 days (d).

contribution of product layer alterations in parallel to charge transfer resistance phenomena. Later on, the magnitude of $|Z|$ slightly drops, whereas the phase angle increases, which can only be attributed to modified product layer morphology and composition, rather than increase in charge transfer. Furthermore, after 7 days' treatment, both magnitude of $|Z|$ and phase angle increase, attributed to improved and more corrosion resistant steel surface (one time constant only at 7 days). The only barrier, rather than "self-healing" effect in specimens CEVn ("empty" vesicles involved) is seen from the gradual decrease of impedance and phase angle and pronounced contribution of a second time constant at the end of the test. These 
observations are in line with the electrochemical behaviour with external polarization - PDP and CVA response. Fig. 5(a) depicts PDP curves for all corroding cases as an overlay of 4-day and 7-day response; Fig. 5(b) presents the response of specimens CEVCn only for all time intervals.

After 4 days of treatment, the corrosion current density for specimens CEn and CEVn are similar (curves 1 and 2, Fig. 5(a)), the anodic current with external polarization for specimens CEVn is even higher than that for CE. This proves the previously discussed diffusion limitations in specimen CEVn and barrier effects of the "empty" vesicles, which do not result in a stable and homogeneous product layer, since anodic currents are not limited but increasing with polarization. Specimens CEVCn present slightly higher corrosion current density, but a significant anodic limitation with external polarization is relevant (curve 3 , Fig. 5(a)). The latter observation is in line with the previously discussed attempts to "repair" initially induced corrosion damage. After 7-day treatment, the CEn specimens (curve 1a, Fig. 5(a)) exhibit the highest anodic and corrosion currents (as expected) and significant shift of corrosion potentials to more cathodic values. In contrast, specimens CEVCn (curve 3a, Fig. 5(a)) present a drop of corrosion and anodic current densities, a shift to more noble corrosion potentials and maintained anodic currents limitations.

After 7-day treatment, specimens CEVn present similar to the 4-day behaviour of active dissolution with external polarization (curves 2a and 2, Fig. 5(a)). The self-healing effect of the "Ca-containing" vesicles, present in the medium of $\mathrm{CE}+$ $\mathrm{NaCl}$ are well seen if a comparison is made of the response for specimens CEVCn with time - Fig. 5(b): after initially low currents (curve 1, $3 \mathrm{~h}$ ), corrosion is initiated (curve 2, $24 \mathrm{~h}$ ); the process is maintained until after 4 days, when slightly higher corrosion current is recorded, most likely corresponding to product layer transformations and Ca-content release and/or nucleation sites effect due to the "Ca-containing" vesicles on the steel surface. The result with prolonged treatment is product layer "repair", evident by a significant drop of corrosion and anodic currents (curve 4, $7 \mathrm{~d}$ ) and further anodic limitations.
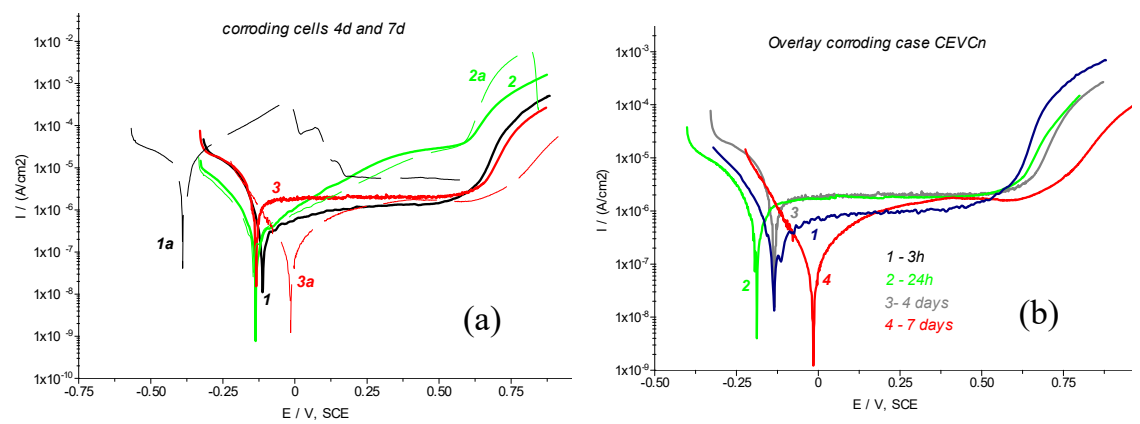

Figure 5: (a) PDP curves for specimens: CEn at $4 \mathrm{~d}$ and $7 \mathrm{~d}$ (curves 1 and 1a); CEVn at $4 \mathrm{~d}$ and $7 \mathrm{~d}$ (curves 2 and 2a); CEVCn at $4 \mathrm{~d}$ and $7 \mathrm{~d}$ (curves 3 and 3a); (b) PDP response for specimen CEVCn at $3 \mathrm{~h}, 24 \mathrm{~h}, 4 \mathrm{~d}$ and $7 \mathrm{~d}$ (curves 1, 2, 3 and 4 respectively). 
CVA tests strongly support all above observations. Fig. 6 presents the 1st, 10th and 20th scans, recorded after treatment in the relevant solutions. As seen from the plots, pitting initiates for specimens CEn and CEVn (at approx. $-325 \mathrm{mV}$ ) with the 1st scan, whereas no pitting was observed for specimens CEVCn, the latter exhibiting the lowest current densities in the reverse scan and most noble re-passivation potential (Fig. 6). After 10 scans, all specimens, including CEVCn show pitting formation at around $-500 \mathrm{mV}$ (Fig. 6) and shift towards higher currents in both anodic and cathodic sweeps. Finally, within 20 scans (Fig. 6), the self-healing effect of the Ca-containing vesicles is evident from the recorded significantly low currents for specimens CEVCn in both anodic and cathodic sweeps, accompanied by the lack of pitting formation. In contrast, for specimens CEn and CEVn, pitting propagation is still relevant, accompanied by already largely increased currents in both anodic and cathodic sweeps.
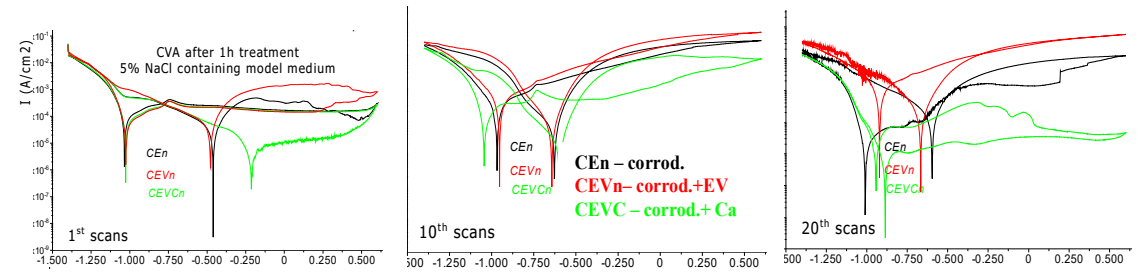

Figure 6: CVA scans for specimens CEn, CEVn and CEVCn.

\subsection{Surface analysis}

The previously discussed possible characteristics, with respect to electrochemical response, of the product layers formed on the steel surface were actually as observed via ESEM, EDX and XPS analysis. Fig. 7 presents ESEM micrographs and the relevant EDX analysis for the corroding cases (b-d); for a comparison, the control case (CE) is depicted in Fig. 7(a) (the layers at higher magnification are given as insets in the EDX patterns).

As it can be seen in Fig. 7, the morphology and composition of the product layer for corroding specimen CEVCn (Fig. 7(d)) resembles the one observed for the control specimen CE (Fig. 7(a)) i.e. a more compact layer, depicting mainly calcium carbonate was observed. It should be noted that for all specimens, $\mathrm{CaCO}_{3}$ was detected by both ESEM/EDX and XPS (Fig. 8) (the XPS and ESEM/EDX investigations were performed after removing the specimens from the model solutions and drying on air). Since the specimens were in immersed condition, carbonation of the solutions during treatment is minimal. Moreover, if a substantial concentration of $\mathrm{CO}_{2}$ in the solutions was present, $\mathrm{FeCO}_{3}$ will form, which would be either detected by XPS and ESEM, or would rather rapidly transform when the specimens are taken on air to more stable iron oxides $\left(\mathrm{FeCO}_{3}\right.$ or "green rust" is not stable in oxygen containing environment). Therefore, the detected surface $\mathrm{CaCO}_{3}$ is a result of the reaction of Ca-containing compounds (as $\mathrm{CaO}$ and $\mathrm{Ca}(\mathrm{OH})_{2}$ previously deposited on the steel surface during treatment) with atm. $\mathrm{CO}_{2}$ upon drying of the specimens on air. Consequently, the presence and 

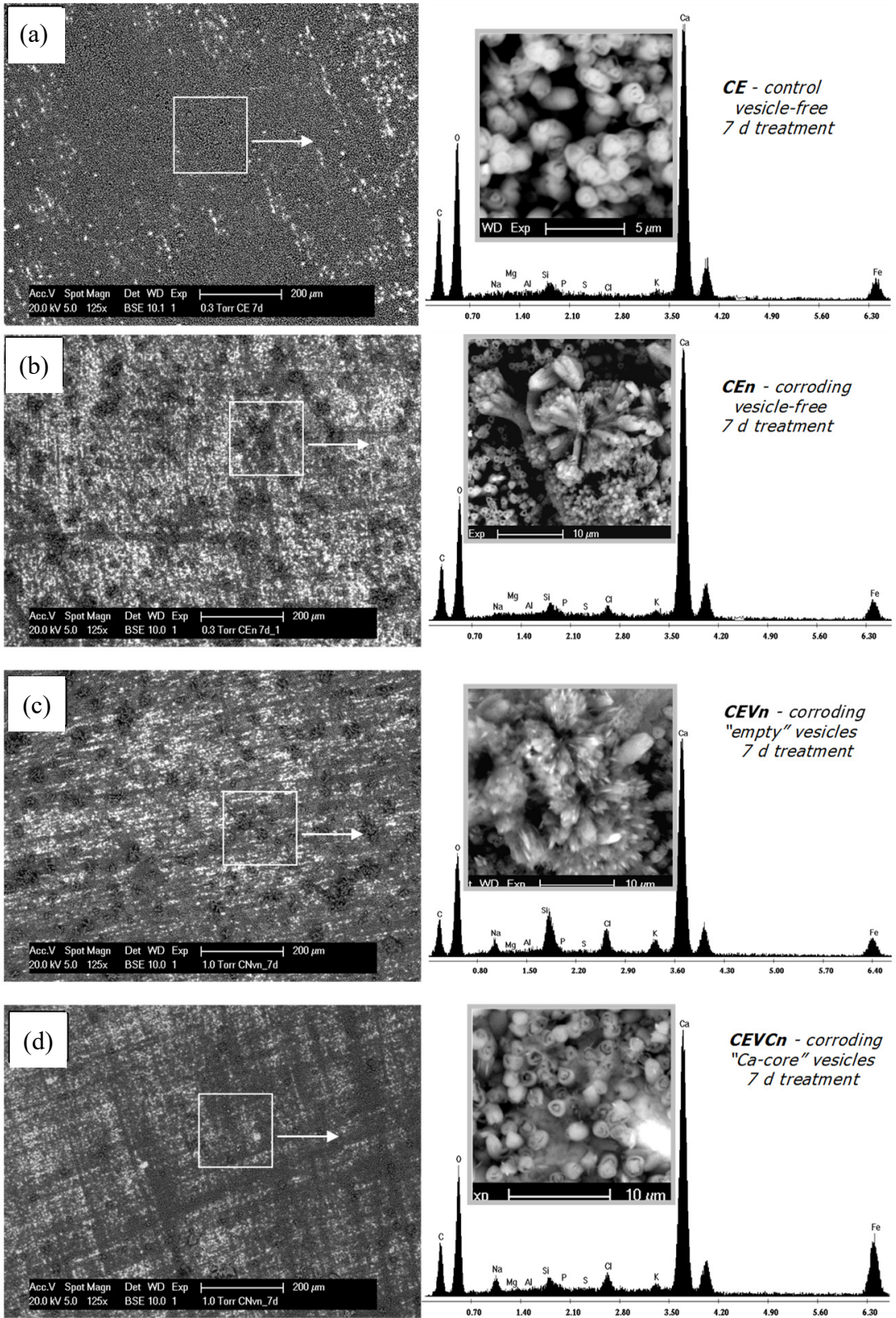

Figure 7: ESEM micrographs and the relevant EDX analysis for control (a) and corroding cases CEn (b), CEVn (c) and CEVCn (d). 
amounts of $\mathrm{CaCO}_{3}$ on the steel surface can serve as an indirect indication for the presence of adhered Ca-containing compounds during treatment in immersed condition. To this end, the control cases and specimens CEVCn present mainly carbonate-rich product layers, whereas for specimens CEn and CEVn (Figs. 7(b), (c)) except $\mathrm{CaCO}_{3}$, chloride-containing iron oxides/hydroxides are relevant. This is in line with the lower corrosion resistance, detected via electrochemical measurements. In specimens CEn and CEVn localised corrosion is basically observed, evidenced by the deposition of voluminous, needle-shape corrosion products (Figs. 7(b), (c)).

An elaborated discussion on the otherwise extensive XPS analysis is outside the scope of this paper and will be separately reported. What needs to be mentioned are only the main observations and features, supporting the above results and discussion.

As previously defined, XPS was performed on the surface of the as-treated specimens (after 7 days) and in depth of the corresponding product layers. Fig. 8 presents the ionizations of $\mathrm{Fe}, \mathrm{O}$ and $\mathrm{C}$ on the "as-treated" surface (scans 1 and 2) and in depth of the products (scans 1' and 2'). With respect to iron oxides and hydroxides, all specimens exhibit a mixture of these with a larger portion of the latter in the outermost sections of the layers (shape and features of the scans 1 and 2 in Fe2p, pronounced peak at $531.6 \mathrm{eV}$ in $\mathrm{O} 1 \mathrm{~s}$, corresponding to $\mathrm{OH}^{-}$) and a larger portion of the former (oxides) in depth of the layers (hematite/magnetite based layers as evident from the characteristic features of Fe2p, $1^{\prime}$ and $2^{\prime}$ and the peaks at lower binding energy $\sim 529 \mathrm{eV}$ in the $\mathrm{O} 1 \mathrm{~s}$ for $1^{\prime}$ and $\left.2^{\prime}\right)$. As also observed, metallic iron appears in depth of the layers as well $(\sim 706 \mathrm{eV})$, which is as expected within product layer removal. The presence of adsorbed in the product layers vesicles for specimen CEVCn is confirmed by the characteristic binding energies in the $\mathrm{C} 1$ s ionizations i.e. $283 \mathrm{eV}$ for PS, $286.5 \mathrm{eV}$ for PEO and $534 \mathrm{eV}$ for PEO
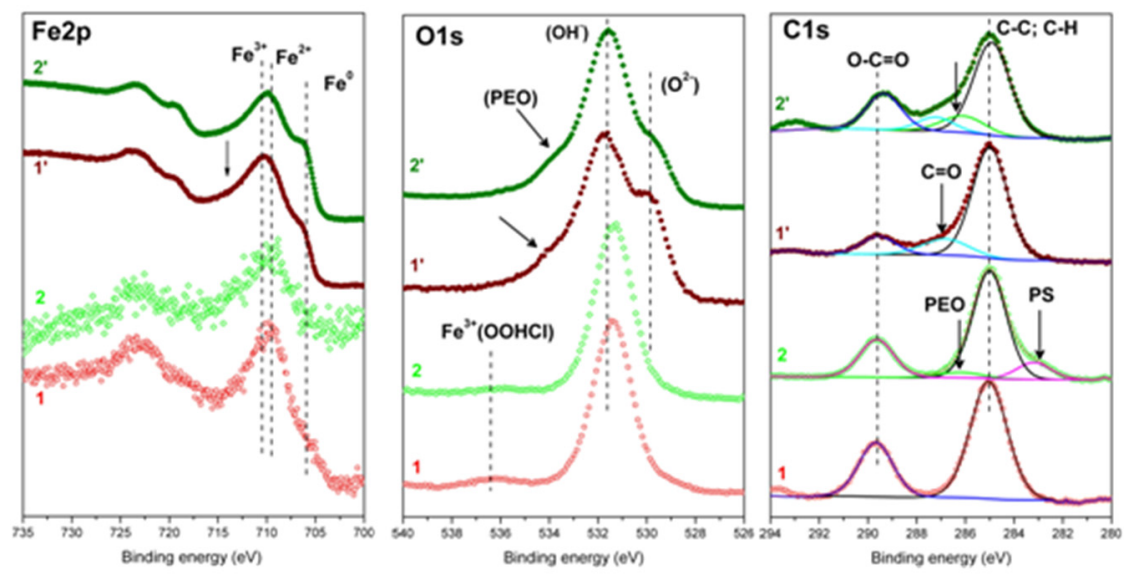

Figure 8: XPS iron (Fe2p), oxygen (O1s) and carbon $(\mathrm{C} 1 \mathrm{~s})$ ionization for specimens CEn (curves 1 and 1') and CEVCn (curves 2 and 2'). 
in the O1s ionizations. Iron oxy/hydroxy chlorides are detected only in the outermost layers for (mainly) specimens CEn (peak at $536.1 \mathrm{eV}$ in O1s), which is actually supported by the morphological observations on the surface of CEn (Fig. 7(b)). In other words, morphological and chemical alterations in the product layers, formed on the steel surface in the presence of Ca-containing vesicles and chloride result in a "self-repair" or "self-healing", evidenced by a more compact and uniform product layer, featuring iron oxy/hydroxides and calcium carbonate only (similar to the layers formed in the control, chloride-free cases). In contrast, for steel treated in vesicles-free or "empty" vesicles containing solutions, voluminous iron-oxy/hydroxy chlorides and lower amount of Ca-containing compounds are present.

\section{Conclusions}

In the framework of a novel approach to corrosion control in reinforced concrete, the application of polymeric nano-particles was investigated. In order to evaluate the difference between "barrier" effects only and self-healing mechanisms, resulting from the "Ca-core" release, the effect of both "empty" and Ca-containing vesicles was studied. In the presence of both vesicle types, corrosion resistance was increased, but the effect was significantly more pronounced when Ca-containing vesicles were involved. The concentration of $0.0024 \mathrm{wt} . \%$ vesicles in the model solutions is minimal and therefore self-repair or self-healing of the product layer solely due to the Ca-component is not realistic. The most plausible mechanism is the nature of incorporation of the Ca-containing vesicles in the product layer, enhanced chloride binding effects and adsorption on active sites on the steel surface. The result is a more uniform and stable steel surface layer, initial pitting formation and propagation, but consecutive healing, as evidenced by surface analysis and electrochemical response i.e. largely reduced anodic and corrosion currents and no further pit propagation when Ca-containing vesicles are present.

\section{References}

[1] Conway, B.E., Theory and Principles of Electrode Processes, The Ronald Press Company: NY, pp. 170-272, 1965.

[2] Broomfield, J., Corrosion of Steel in Concrete, Understanding, Investigating and Repair, Spon: London, 1997.

[3] Şahmaran, M., Effect of flexure induced transverse crack and self-healing on chloride diffusivity of reinforced mortar. Journal of Materials Science, 42(22), pp. 9131-9136, 2007.

[4] Elsener, B., Macrocell corrosion of steel in concrete - implications for corrosion monitoring. Cement and Concrete Composites, 24(1), pp. 65-72, 2002.

[5] Garces, A., Andrade, M.C., Saez, A. \& Alonso, M.C., Corrosion of reinforcing steel in neutral and acid solutions simulating the electrolytic 
environments in the micropores of concrete in the propagation period. Corrosion Science, 47(2), pp. 289-306, 2005.

[6] Broomfield, J.P., Corrosion of steel in concrete. Uhlig's Corrosion Handbook, ed. R.W. Revie, Wiley: Hoboken, NJ, pp. 633-648, 2011.

[7] Bertolini, L., Elsener, B., Pedeferri, P. \& Polder, R., Corrosion of Steel in Concrete: Prevention, Diagnosis, Repair, 1st ed., Wiley: Weinheim, 2004.

[8] Petterson, K., Chloride threshold value and the corrosion rate in reinforced concrete. Corrosion and Corrosion Protection of Steel in Concrete, vol. 1, ed. R.N. Swamy, Sheffield Academic Press: Sheffield, p. 461, 1994.

[9] Pedeferri, P., Cathodic protection and cathodic prevention. Construction and Building Materials, 10(5), pp. 391-402, 1996.

[10] Bassi, R. \& Davies, H., Testing anti-carbonation coatings for concrete, BRE Information paper IP7/96, 1996.

[11] Buenfeld, N.R., \& Zhang, J.-Z., Chloride diffusion through surface-treated mortar specimens. Cement and Concrete Research, 28(5), pp. 665-674, 1998.

[12] Castle, J.E., Cathodic disbondment. AIP Conference Proceedings, 354, pp. 432-449, 1996.

[13] Bird, C.E, Bond of galvanized steel reinforcement in concrete. Nature, 194, p. 798, 1962.

[14] Herrera, M.J., Martinez, M. \& Perez, J.T., Evaluation of electrochemical behavior of two inhibitors for galvanized rebars in alkaline solutions, paper 831, 210th ECS meeting, Cancun, MX, 2006.

[15] International Lead Zinc Research Organisation, Galvanized reinforcement for concrete II. Res., Triangle Park NC, USA, 1981.

[16] Kayali, O. \& Yeomans, S.R., Bond of ribbed galvanized reinforcing steel in concrete. Cement and Concrete Composites, 22(6), pp. 459-467, 2000.

[17] Andrade, C. \& Holst, J.D., Coating Protection for Reinforcement, Comite Euro-International Du Beton, State of the Art Report, Thomas Telford Publication: London, p. 51, 1995.

[18] Mietz, J., Electrochemical Rehabilitation Methods for Reinforced Concrete Structures: A State of the Art Report, Institute of Materials, London, UK, 1998.

[19] Andrade, C., Keddam, M., Nóvoa, X.R., Pérez, M.C., Rangel, C.M. \& Takenouti, H., Electrochemical behaviour of steel rebars in concrete: influence of environmental factors and cement chemistry. Electrochimica Acta, 46(24-25), pp. 3905-3912, 2001.

[20] Gaidis, M., Chemistry of corrosion inhibitors. Cement and Concrete Composites, 26(3), pp. 181-189, 2004.

[21] Wombacher, F., Maeder, U. \& Marazzani, B., Aminoalcohol based mixed corrosion inhibitors. Cement and Concrete Composites, 26(3), pp. 209-216, 2004.

[22] Koleva, D.A., van Breugel, K., Ye, G., Zhou, J., Chamululu, G. \& Koenders, E.A.B., Porosity and permeability of mortar specimens incorporating PEO113-b-PS218 micelles. ACI Materials Journal, 267, pp. 101-110, 2009. 
[23] Koleva, D.A., Hu, J., van Breugel, K., Milkova, V. \& Petrov, P., The influence of tailored nano/micro polymeric aggregates on material properties of cement-based systems. Materials, Methods and Technologies, 5(1), pp. 63-73, 2011.

[24] Hu, J., Koleva, D.A., Petrov, P. \& van Breugel, K., Polymeric vesicles for corrosion control in reinforced mortar: Electrochemical behavior, steel surface analysis and bulk matrix properties. Corrosion Science, 65, pp. 414-430, 2012.

[25] Hu, J., Koleva, D.A., Ma, Y., Schlangen, E., Petrov, P. \& van Breugel, K., The influence of admixed micelles on the microstructural properties and global performance of cement-based materials. Cement and Concrete Research, 42(8), pp. 1122-1133, 2012.

[26] Hu, J., Koleva, D.A. \& van Breugel, K., Corrosion performance of reinforced mortar in the presence of polymeric nano-aggregates: electrochemical behavior, surface analysis, and properties of the steel/cement paste interface. Journal of Materials Science, 47(12), pp. 4981-4995, 2012.

[27] Hu, J., Koleva, D.A., de Wit, J.H.W., Kolev, H. \& van Breugel, K., Corrosion performance of carbon steel in simulated pore solution in the presence of micelles. Journal of the Electrochemical Society, 158(3), pp. C76-C87, 2011.

[28] Hu, J., Koleva, D.A. \& van Breugel, K., The influence of $\mathrm{PEO}_{113}$-b-PS $\mathrm{PS}_{780^{\circ}}$ vesicles on the corrosion performance of carbon steel in simulated pore solution. ECS Transactions, 41(16), pp. 1-9, 2012. 